\title{
Extended Fibonacci Cubes
}

\author{
Jie Wu, Senior Member, IEEE
}

\begin{abstract}
The Fibonacci Cube is an interconnection network that possesses many desirable properties that are important in network design and application. The Fibonacci Cube can efficiently emulate many hypercube algorithms and uses fewer links than the comparable hypercube, while its size does not increase as fast as the hypercube. However, most Fibonacci Cubes (more than $2 / 3$ of all) are not Hamiltonian. In this paper, we propose an Extended Fibonacci Cube $\left(E F C_{1}\right)$ with an even number of nodes. It is defined based on the same sequence $F(i)=F(i-1)+F(i-2)$ as the regular Fibonacci sequence; however, its initial conditions are different. We show that the Extended Fibonacci Cube includes the Fibonacci Cube as a subgraph and maintains its sparsity property. In addition, it is Hamiltonian and is better in emulating other topologies. Specifically, the Extended Fibonacci Cube can embed binary trees more efficiently than the regular Fibonacci Cube and is almost as efficient as the hypercube, even though the Extended Fibonacci Cube is a much sparser network than the hypercube. We also propose a series of Extended Fibonacci Cubes with even number of nodes. Any Extended Fibonacci Cube $\left(E F C_{k}\right.$, with $\left.k \geq 1\right)$ in the series contains the node set of any other cube that precedes $E F C_{k}$ in the series. We show that any Extended Fibonacci Cube maintains virtually all the desirable properties of the Fibonacci Cube. The $E F C_{k} s$ can be considered as flexible versions of incomplete hypercubes, which eliminates their restriction on the number of nodes, and, thus, makes it possible to construct parallel machines with arbitrary sizes.
\end{abstract}

Index Terms-Fibonacci numbers, Hamiltonian graphs, graph embedding, hypercubes, interconnection topologies.

\section{INTRODUCTION}

A widely studied interconnection topology is the hypercube [13]. The hypercube provides a rich interconnection structure which permits many other topologies to be efficiently emulated. Numerous research projects have been undertaken related to hypercube design aspects and hypercube applications [7]. These have resulted in several research prototypes and commercial products [3].

Unfortunately, the number of nodes $2^{n}$ in an $n$-dimensional hypercube $Q(n)$ grows rapidly as $n$ increases. This limits considerably the choice of the number of nodes in the graph. The Fibonacci Cube (FC) proposed by Hsu [10] is a special subcube of a hypercube based on Fibonacci numbers [6]. It has been shown that the Fibonacci Cube can efficiently emulate many hypercube algorithms. Fibonacci Cubes use fewer links than comparable hypercubes and their size does not increase as fast as hypercubes. The structural analysis of the Fibonacci Cube has been extensively studied in [2] and its applications in [9]. A Fibonacci Cube can also be viewed as resulting from a complete hypercube after some nodes become faulty and the system is reconfigured. Therefore, the Fibonacci Cube not only allows the construction of systems of arbitrary sizes, but also exposes the nature of hypercube systems operating in a gracefully degraded mode.

Most Fibonacci Cubes are not Hamiltonian. In fact, Cong et al. [5] showed that less than $1 / 3$ of Fibonacci Cubes are Hamiltonian. The challenge here is to define a new graph that satisfies the above mentioned properties, while still maintaining all the desirable properties of the Fibonacci

- J. Wu is with the Department of Computer Science and Engineering, Florida Atlantic University, Boca Raton, FL 33431.

E-mail: jie@cse.fau.edu.

Manuscript received 14 July 1994

For information on obtaining reprints of this article, please send e-mail to: tpds@computer.org, and reference IEEECS Log Number 100911.
Cube. Moreover, the new graph should be sparse; at least, it should have a similar degree of sparsity as the Fibonacci Cube.

In this paper, we propose the Extended Fibonacci Cube $\left(E F C_{1}\right)$, which is based on the same sequence $F(i)=F(i-1)+$ $F(i-2)$ as the Fibonacci Cube; however, its initial conditions are defined differently. More specifically, in $E F C_{1}$, the two initial values are 2 and 4 . Therefore, $F(i)$ is always an even number. We show that $E F C_{1}$ is Hamiltonian and maintains virtually all the desirable properties of $F C$. Our results also show that $E F C_{1}$ is superior to $F C$ in terms of various structural properties.

We then propose a series of Extended Fibonacci Cubes in such a way that any Extended Fibonacci Cube $\left(E F C_{k}\right.$, with $k \geq 1$ ) in the series contains the node set of any other cube that precedes $E F C_{k}$. The $E F C_{k}$ s can be considered as flexible versions of incomplete hypercubes, which eliminates their restriction on the number of nodes, and thus, makes it possible to construct parallel machines with arbitrary sizes. Moreover, all the cubes in the series are Hamiltonian, and they are better in terms of emulating other topologies (i.e., embedding) than regular Fibonacci Cubes. We show that each cube in the series has a distinct size, and each cube maintains virtually all the desirable properties of the Fibonacci Cube.

Our study focuses on the structural properties of the Extended Fibonacci Cube. The use of the Extended Fibonacci Cube in various applications is beyond the scope of this paper. In summary, the structural advantage of the Extended Fibonacci Cube over the regular Fibonacci Cube manifests itself in three ways:

1) The series of Extended Fibonacci Cube allows more choices to construct systems of different sizes.

2) All Extended Fibonacci Cubes are Hamiltonian, while less than $1 / 3$ of Fibonacci Cubes are Hamiltonian. 
3) The Extended Fibonacci Cube is better than the regular Fibonacci Cube in emulating other topologies, such as hypercubes.

Throughout this paper, except for a few important theorems, proofs for theorems and other details are not included. The reader may refer to [15] for details.

\section{Extended Fibonacci Cubes}

As mentioned earlier, Extended Fibonacci Cubes are defined based on the same Fibonacci sequence. However, their initial conditions are different. The following defines the first Extended Fibonacci Cube in the series. The symbol \| denotes a concatenation operation; for example, $01 \|\{0,1\}=$ $\{010,011\}$ and $01 \|\{\}=01$.

DEFINITION 1. Assume $\operatorname{EFC}_{1}(n)=\left(V_{1}(n), E_{1}(n)\right), \operatorname{EFC}_{1}(n-1)=$ $\left(V_{1}(n-1), E_{1}(n-1)\right)$, and $\operatorname{EFC}_{1}(n-2)=\left(V_{1}(n-2)\right.$, $\left.E_{1}(n-2)\right)$. Then $V_{1}(n)=0\left\|V_{1}(n-1) \cup 10\right\| V_{1}(n-2)$. Two nodes in $\operatorname{EFC}_{1}(n)$ are connected by an edge in $E_{1}(n)$ if and only if their labels differ in exactly one bit position. As initial conditions for recursion, $V_{1}(3)=\{0,1\}$ and $V_{1}(4)=\{00,10,11,01\}$.

Fig. 1 shows examples of $E F C_{1}$ of size $n$, where $n=3,4,5,6$, respectively. An $E F C_{1}$ of size $n$ consists of one $E F C_{1}$ of size $n-1$ and one $E F C_{1}$ of size $n-2$.

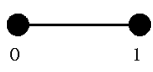

(a)

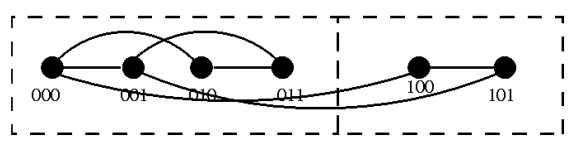

(c)

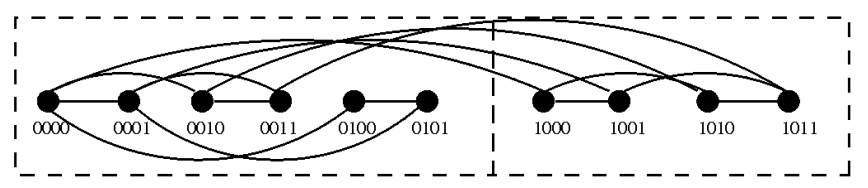

(d)

Fig. 1. Extended Fibonacci Cubes $E F C_{1}$ s: (a) $E F C_{1}$ (3), (b) $E F C_{1}(4)$, (c) $E F C_{1}(5)$, and (d) $E F C_{1}(6)$.

THEOREM 1. For any $n \geq 4, E F C_{1}(n)$ is a Hamiltonian graph.

PROOF. We prove this theorem by induction on $n$. In addition, we show that, for each $E_{F C}(n), n \geq 4$, a Hamiltonian path of type $000^{n-4} \rightarrow 100^{n-4} \rightarrow G \rightarrow 010^{n-4}$ can be constructed, where $0^{n-4}$ is a sequence of 0 of length $n-4$ (could be empty). $G$ is a sequence of adjacent nodes, and the first and last nodes of $G$ are adjacent to $100^{n-4}$ and $010^{n-4}$, respectively. Since the first and last nodes of the Hamiltonian path are adjacent, this
Hamiltonian path is also a Hamiltonian cycle. In addition, the code for $\operatorname{EFC}_{1}(n)$ is one bit longer than the one for $\operatorname{EFC}_{1}(n-1)$. For $n=4$, it is obviously true and the Hamiltonian path in $E F C_{1}$ is $00 \rightarrow 10 \rightarrow \underset{G}{11} \rightarrow 01$. For $n=5$, the Hamiltonian path in $E F C_{1}(5)$ is $000 \rightarrow 100 \rightarrow \underbrace{101 \rightarrow 001 \rightarrow 011}_{G} \rightarrow 010$. Assume this theorem holds for all the $\operatorname{EFC}_{1}(n)$ s, such that $n$ $<k$ where $k \geq 4$. Based on the induction assumption, we have a Hamiltonian path $000^{k-5} \rightarrow 100^{k-5} \rightarrow G_{1} \rightarrow 010^{k-5}$ for $\operatorname{EFC}_{1}(k-1)$ and $000^{k-6} \rightarrow 100^{k-6} \rightarrow G_{2} \rightarrow 010^{k-6}$ for $E F C_{1}(k-2)$. We construct a Hamiltonian cycle for $E F C_{1}(k)$ as follows:

$$
\begin{aligned}
& 0 \| 000^{k-5} \rightarrow 10|| 000^{k-6} \rightarrow \\
& \underbrace{10\left\|010^{k-6} \rightarrow 10\right\| G_{2}^{-1} \rightarrow 10\left\|100^{k-6} \rightarrow 0\right\| 010^{k-5} \rightarrow 0 \| G_{1}^{-1}}_{G} \rightarrow \\
& 0 \mid 100^{k-5},
\end{aligned}
$$

where $G^{-1}$ is the reverse sequence of $G$.

Recall that the Fibonacci Cube is defined as follows [10]: Assume $F C(n)=(V(n), E(n)), F C(n-1)=(V(n-1), E(n-1))$, and $F C(n-2)=(V(n-2), E(n-2))$. Then, $V(n)=0 \| V(n-1)$ $\cup 10 \| V(n-2)$. Two nodes in $F C(n)$ are connected by an edge in $E(n)$ if and only if their labels differ in exactly one bit position. As initial conditions for recursion, $V(2)=\{\}$, $V(3)=\{0,1\}$. Cong et al. [5] showed that less than $1 / 3$ of Fibonacci Cubes are Hamiltonian. Therefore, the proposed Extended Fibonacci Cube is better in emulating rings than the regular Fibonacci Cube. The relationship between FC and $E F C_{1}$ is revealed in the following theorem.

THEOREM 2. FC $(n)$ is a proper subgraph of $\operatorname{EFC}_{1}(n)$, for $n \geq 4$.

PROOF. Since, in both $E F C_{1}$ and $F C$, nodes are connected if their labels differ in exactly one bit position, it suffices to show that $V(n) \subset V_{1}(n)$, where $V(n)$ and $V_{1}(n)$ are node sets of $F C(n)$ and $\operatorname{EFC}_{1}(n)$, respectively. We prove this theorem by induction on $n$. Clearly, $V(4) \subset V_{1}(4)$ and $V(5) \subset V_{1}(5)$. Assume $V(n) \subset$ $V_{1}(n)$ for all $n<k$. When $n=k>6$, based on the definition of $V(n)=0\|V(n-1) \cup 10\| V(n-2)$, we have

$$
V(k)=0\|V(k-1) \cup 10\| V(k-2) \subset 0 \| V_{1}(k-1)
$$

$$
\cup 10 \| V_{1}(k-2)=V_{1}(k) \text {. }
$$

A path between two nodes is called Hamming distance path if the length of this path is equal to the Hamming distance of these two nodes.

THEOREM 3. There exists a Hamming distance path for any two nodes in $E_{1} C_{1}(n)$.

ProOF. We prove this theorem by induction on $n$. Clearly, this theorem holds for $n=3,4,5$. Assume that this theorem holds for all $n<k$. When $n=k$ and $V_{1}(k)=$ 
$0\left\|V_{1}(k-1) \cup 10\right\| V_{1}(k-2)$, randomly select two nodes; if both belong to $0 \| V_{1}(k-1)$ (or $10 \| V_{1}(k-2)$ ), this theorem holds based on the induction assumption. We only need to consider cases when one node is in $0 \| V_{1}(k-1)$ and the other is in $10 \| V_{1}(k-2)$. Because $0\left\|V_{1}(k-1)=00\right\| V_{1}(k-2) \cup 010 \| V_{1}(k-3)$, the problem is divided into:

1) proving there is a Hamming distance path between a node in $10 \| V_{1}(k-2)$ and a node in $00 \| V_{1}(k-2)$, and

2) proving there is a Hamming distance path between a node in $10 \| V_{1}(k-2)$ and a node in $010 \| V_{1}(k-3)$.

Case 1 is obvious, since, based on the induction assumption, there exists a Hamming distance path between any two nodes in $V_{1}(k-2)$. Case 2 can be proved by using the fact that $010 \| V_{1}(k-3) \subset 01 V_{1}(k-2)$. Then, the problem is reduced to find a Hamming distance path from a node in $01 \| V_{1}(k-2)$, via $00 \| V_{1}(k-2)$, to a node in $10 \| V_{1}(k-2)$.

In the subsequent discussion, the $\|$ notation will be omitted without causing confusion. For example, 01\|10 will be written as 0110 .

COROLLARY. The diameter of $E_{F}(n)$ is $n-2$.

THEOREM 4. The node degree of a node in $\operatorname{EFC}_{1}(n)$ is between $\left\lceil\frac{n}{3}\right\rceil$ and $n-2$.

PROOF. Since there are $n-2$ bits in each node of $E_{F C}(n)$, based on [10], $n-2$ is the maximum node degree of $F C(n)$, the maximum node degree of $E_{F} C_{1}(n)$ is $n-2$. Let $\tau_{n}$ denote the minimum node degree in $\operatorname{EFC}_{1}(n)$. Based on the definition of $E F C_{1}(n)$,

$V_{1}(n)=00 V_{1}(n-2) \cup 010 V_{1}(n-3) \cup 10 V_{1}(n-2)$.

We have,

$$
\begin{aligned}
& \min \left\{\tau_{n-2}+1, \tau_{n-3}+1, \tau_{n-2}+1\right\} \leq \tau_{n} \leq \\
& \min \left\{\tau_{n-2}+2, \tau_{n-3}+1, \tau_{n-2}+1\right\} .
\end{aligned}
$$

Since $\tau_{n-2} \geq \tau_{n-3}$, we have $\tau_{n}=\tau_{n-3}+1$. Based on $\tau_{3}=$ $1, \tau_{4}=2, \tau_{5}=2$, we have $\tau_{n}=\left\lceil\frac{n}{3}\right\rceil$.

\section{Extensions}

In this section, we consider another Extended Fibonacci Cube, called $\operatorname{EFC}_{2}(n)$, by changing the initial conditions of the Fibonacci sequence. We show that $E_{F} C_{1}(n)$ is a proper subgraph of $\operatorname{EFC}_{2}(n)$, where $n \geq 5$. However, $\operatorname{EFC}_{2}(n)$ still maintains the sparsity of $F C(n)$ and virtually all the other desirable properties. We show that the sequences generated by $F C, E F C_{1}$, and $E F C_{2}$ are mutually disjoint and propose guidelines for constructing different Extended Fibonacci Cubes with their corresponding sequences mutually disjoint. Because these Extended Fibonacci Cubes have similar properties, we can use them as a group (probably together with $F C$, although most of $F C^{\prime}$ s are not Hamiltonian) to construct a special series of incomplete hypercubes [11], [14]. In this way, the restriction on the number of nodes in regular hypercubes $\left(2^{n}\right)$ is further relaxed or totally removed.
DEFINITION 2. Assume $\operatorname{EFC}_{2}(n)=\left(V_{2}(n), E_{2}(n)\right), \operatorname{EFC}_{2}(n-1)=$ $\left(V_{2}(n-1), E_{2}(n-1)\right)$, and $\operatorname{EFC}_{2}(n-2)=\left(V_{2}(n-2)\right.$, $\left.E_{2}(n-2)\right)$. Then $V_{2}(n)=0\left\|V_{2}(n-1) \cup 10\right\| V_{2}(n-2)$. Two nodes in $\operatorname{EFC}_{2}(n)$ are connected by an edge in $E_{2}(n)$ if and only if their labels differ in exactly one bit position. As initial conditions for recursion, $V_{2}(4)=\{00,10,11$, $01\}$ and $V_{2}(5)=\{000,100,101,111,110,010,011,001\}$.

Following the proofs used in the previous section, we can prove that Theorems 1 to 3 are still valid. That is, $E F C_{2}$ is Hamiltonian and the diameter of $E F C_{2}(\mathrm{n})$ is $n-2$.

THEOREM 5. $\operatorname{EFC}_{1}(n)$ is a proper subgraph of $E F C_{2}(n)$, for $n \geq 6$.

DEFINITION 3. A series of Extended Fibonacci Cubes is defined as $\left\{E F C_{k}, k \geq 1\right\}$, where $\operatorname{EFC}_{k}(n)=\left\{V_{k}(n), E_{k}(n)\right\}, \operatorname{EFC}_{k}(n-1)=$ $\left(V_{k}(n-1), E_{k}(n-1)\right)$, and $\operatorname{EFC}_{k}(n-2)=\left(V_{k}(n-2)\right.$, $\left.E_{k}(n-2)\right)$. Then $V_{k}(n)=0\left\|V_{k}(n-1) \cup 10\right\| V_{k}(n-2)$. Two nodes in $E_{F C}(n)$ are connected by an edge in $E_{k}(n)$ if and only if their labels differ in exactly one bit position. As initial conditions for recursion, $V_{k}(k+2)=\{\underbrace{d d \ldots d d}_{k}\}$ and $V_{k}(k+3)=\{\underbrace{d d \ldots d d}_{k+1}\}$, where $d$ is either 1 or 0.

THEOREM 6. For any $n$, such that $n \geq i+3$ and $n \geq j+3$, $\operatorname{EFC}_{i}(n)$ is a proper subgraph of $\operatorname{EFC}_{j}(n)$ if $i<j$.

Based on the result of Theorem 6, we represent the relationship among regular hypercubes, Fibonacci Cubes, and Extended Fibonacci Cubes in the series in Fig. 2, where the fact that a graph $G$ is a subgraph of another graph $H$ is represented by a rectangle (representing graph $G$ ) nested within another rectangle (representing graph $H)$. $Q(n)$ denotes an $n$-dimensional hypercube.

By using the series of Extended Fibonacci Cubes defined in Definition 3, the rigid restriction of the hypercube topology can be removed. For example, if we want to construct a cube consisting of 10 to 100 nodes, there are only three options for regular hypercube structures, that is, 16, 32, 64 . Within the series of Fibonacci Cubes (including $F C, E F C_{k}$ with $k \leq 5$ ) there are 18 choices (see Table 1 ). They are: 10 , $12,13,20,21,26,32,34,42,48,52,55,64,68,80,84,89$, 96. In Table 1 , the element at the $k$ th row and the $n$th column is the size of $F_{k}(n)$. For example, the element at the second row (the row for $F_{2}(n)$ ) and the fifth column is $F_{2}(5)=8$. For each row that represents an $E F C$, two initial values are placed in two square boxes.

It is easy to verify that Theorem 3 still holds for any $E F C_{k}$ and the diameter is $n-2$ for any $E F C_{k}$. Moreover, we have the following results for node degree in $E F C_{k}$.

THEOREM 7. The node degree of a node in $\operatorname{EFC}_{k}(n)$ is between $\left\lceil\frac{n-(k-1)}{3}\right\rceil+(k-1)$ and $n-2$.

In Theorem $7, k$ is the sequence number in the series of Extended Fibonacci Cubes and $n$ is the dimension of the cube. When $k$ is closer to $n$, the resultant $E F C_{k}$ resembles more to the regular hypercube. Theorem 7 shows that the minimum node degree of the corresponding $E F C_{k}$ increases as $k$ increases. The proof can be done in a similar way used in proving Theorem 4. 


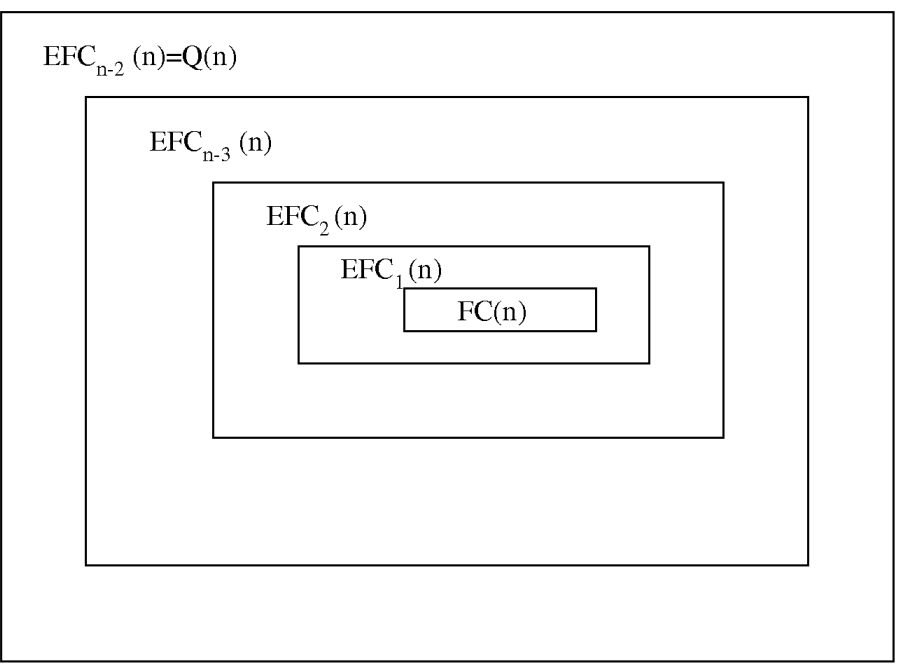

Fig. 2. Relationship among hypercubes, Fibonacci Cubes, and Extended Fibonacci Cubes.

TABLE 1

SEQUENCES FOR $F C, E F C_{1}$, AND $E F C_{2}$, ETC.

\begin{tabular}{||l||c|c|c|c|c|c|c|c|c|c|c|c|c||}
\hline dimension $(n)$ & 0 & 1 & 2 & 3 & 4 & 5 & 6 & 7 & 8 & 9 & 10 & 11 & 12 \\
\hline \hline$F(n)$ & 0 & 11 & 1 & 2 & 3 & 5 & 8 & 13 & 21 & 34 & 55 & 89 & 144 \\
\hline$F_{1}(n)$ & - & - & - & 2 & 4 & 6 & 10 & 16 & 26 & 42 & 68 & 110 & 178 \\
\hline$F_{2}(n)$ & - & - & - & - & 4 & 8 & 12 & 20 & 32 & 52 & 84 & 136 & 220 \\
\hline$F_{3}(n)$ & - & - & - & - & - & 8 & 16 & 24 & 40 & 64 & 104 & 168 & 272 \\
\hline$F_{4}(n)$ & - & - & - & - & - & - & 16 & 32 & 48 & 80 & 128 & 208 & 336 \\
\hline$F_{5}(n)$ & - & - & - & - & - & - & - & 32 & 64 & 96 & 160 & 256 & 416 \\
\hline$F_{6}(n)$ & - & - & - & - & - & - & - & - & 64 & 128 & 192 & 320 & 512 \\
\hline
\end{tabular}

Let $F(n), F_{1}(n)$, and $F_{2}(n)$ denote the size of $F C(n), \operatorname{EFC}_{1}(n)$, and $\operatorname{EFC}_{2}(n)$, respectively. The following theorem shows that all these numbers are distinct for different selections of $n$.

THEOREM 8. Except for initial conditions, the sequences generated by $F C, E F C_{1}$, and $E F C_{2}$ are mutually disjoint.

PROOF. We prove this theorem by contradiction. Consider two Fibonacci sequences $F_{i}$ and $F_{j}$ with different initial conditions. Suppose $F_{i}(n)=F_{j}(m)$, where $n$ is the smallest number for $F_{i}$ satisfying this condition. Based on the definition of the Fibonacci sequence, we have

$$
F_{i}(n-1)+F_{i}(n-2)=F_{j}(m-1)+F_{j}(m-2)
$$

Since $n$ is the smallest number for $F_{i}$ satisfying the equality condition, we have

$$
\begin{aligned}
& F_{i}(n-1)>F_{j}(m-1) \\
& F_{i}(n-2)<F_{j}(m-2)
\end{aligned}
$$

or

$$
F_{i}(n-1)<F_{j}(m-1)
$$

$$
F_{i}(n-2)>F_{j}(m-2)
$$

From (1) and the definition of Fibonacci sequence, $F(n-1)=F(n-2)+F(n-3)$, we have

$$
F_{i}(n-2)+F_{i}(n-3)>F_{j}(m-2)+F_{j}(m-3)
$$

From (5) and (2), we have

$$
F_{i}(n-3)>F_{j}(m-3) \text {. }
$$

Similarly, from (3) and (4), we have

$$
F_{i}(n-3)<F_{j}(m-3) .
$$

It is easy to prove by induction that, for any $p<n$, there exists $q$ such that,

$$
\begin{gathered}
F_{i}(p)>F_{j}(q) \\
F_{i}(p-1)<F_{j}(q-1)
\end{gathered}
$$

or

$$
F_{i}(p)<F_{j}(q)
$$

$$
F_{i}(p-1)>F_{j}(q-1)
$$




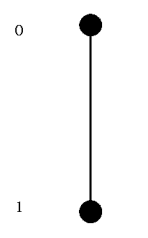

(a)

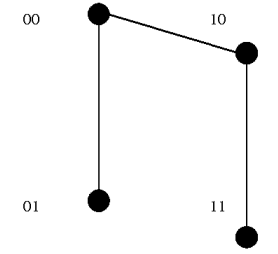

(b)

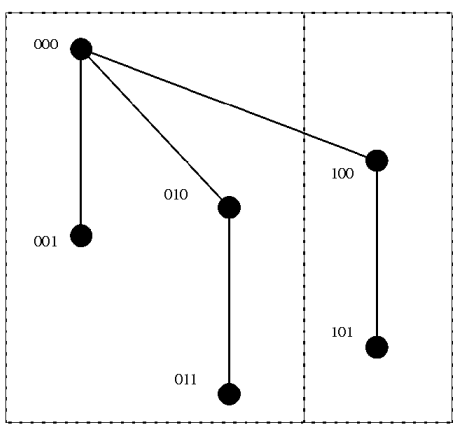

(c)

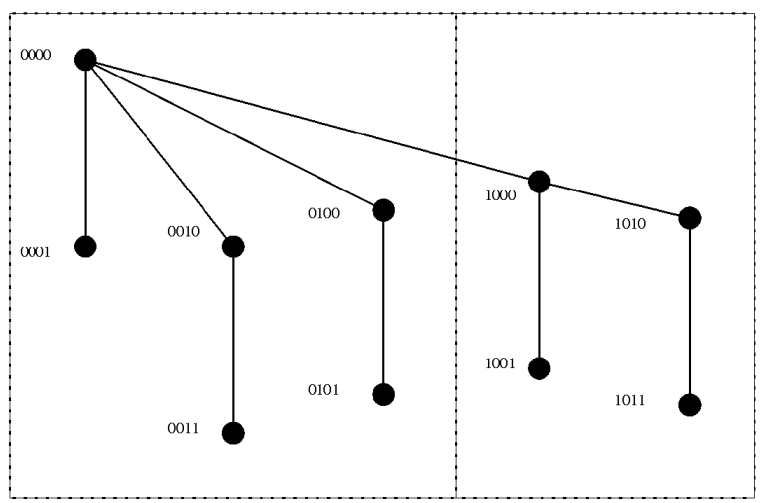

(d)

Fig. 3. Extended Fibonacci Trees: (a) $T_{1}$ (3), (b) $T_{1}$ (4), (c) $T_{1}(5)$, and (d) $T_{1}(6)$.

It is easy to see from Table 1 (where the numbers in boxes are initial conditions) that, for $n \leq 7$, except for numbers that correspond to initial conditions, all the numbers from different sequences are distinct. Also, for all $q$,

$$
F_{1}(6)>F_{2}(q-1) \wedge F_{1}(7)>F_{2}(q)
$$

or

$$
F_{1}(6)<F_{2}(q-1) \wedge F_{1}(7)<F_{2}(q) .
$$

Each of the above cases violates necessary conditions (6) and (7) (or (8) and (9)) for the equality condition. That is, there do not exist $m$ and $n$, such that $F_{i}(m)=F_{j}(n)$. Similar reasoning can be used for $F_{1}$ and $F$, and for $F_{2}$ and $F$.

CONJECTURE. Except for initial conditions, the sequences generated by $F C, E F C_{k}$, for $k \geq 1$, are mutually disjoint.

The validation of this conjecture can be easily seen from Table 1 for small dimensions. Tests have been conducted to show that the above conjecture holds for $F C \cup\left\{E F C_{k}, 1 \leq k \leq\right.$ $20\}$. The significance of the above disjoint property is the following: by considering Fibonacci cube-based systems as incomplete hypercubes, resulting from a complete hypercube after some nodes become faulty and systems are reconfigured. The Extended Fibonacci Cube is more flexible than the regular Fibonacci Cube, since it provides more choices of systems with difference sizes.

\section{Extended Fibonacci Trees}

In this section, we study the Extended Fibonacci Tree $\left(T_{1}\right)$, which is a special spanning tree of the Extended Fibonacci Cube $\left(E F C_{1}\right)$. The extension for general Extended Fibonacci Cubes $\left(E F C_{k}\right)$ is straightforward. We then study an application that uses this tree structure.

DEFINITION 4. The Extended Fibonacci Tree $T_{1}(n)$ of $E F C_{1}(n)$ is defined as follows: (Base) $T_{1}(3)$ and $T_{1}(4)$ are defined as shown in Fig. $2 a$ and $2 b$. Basically, $T_{1}(3)$ is $E F C_{1}(3)$ with node 0 being the root and $T_{1}(4)$ is an $E F C_{1}(4)$ rooted at node 00 after removing the link connecting nodes 01 and 11. (Recursion) $T_{1}(n)(n>4)$ consists of $T_{1}(n-1)$ and $T_{1}(n-2)$ by connecting the root of $T_{1}(n-2)$ as a child of the root of $T_{1}(n-1)$. Suppose $T_{1}(n)$ also denotes the set of nodes in $T_{1}(n)$, then $T_{1}(n)=0\left\|T_{1}(n-1) \cup 10\right\| T_{1}(n-2)$.

Fig. 3 shows the structure of Extended Fibonacci Trees $T_{1}(n)$, for $n=3,4,5,6$. Clearly, $T_{1}(n)$ has the same node set as $\operatorname{EFC}_{1}(n)$. Hence, $T_{1}(n)$ is a spanning tree of $E F C_{1}(n)$. Extended Fibonacci Trees can be used to implement several tree-based algorithms efficiently. As an example, we show the implementation of two general procedures: tree contraction and tree expansion. A tree contraction is used to reduce a rooted tree to its root by a sequence of conflictfree operations to remove its vertices. Tree expansion is an inverse operation of tree contraction. By conflict-free, we mean that two vertices removed (or added) in the same 
time step do not have a parent-child relation and that, at most, one of a node's child is removed (or added) at a time. It has been shown [1] that tree contraction and expansion are general techniques for scheduling parallel computations on tree. For example, the prefix computation problem can be converted to a tree contraction and expansion problem.

The following are tree contraction and expansion algorithms for $T_{1}(n)$, which are executed at each node $a$ of $\operatorname{EFC}_{1}(n) . a(i)$ denotes the $i$ th bit of node $a$ (counting from the right), and node $a^{i}$, the neighbor of node $a$, along dimension $i$, i.e., nodes $a^{i}$ and $a$ differ only in the $i$ th bit.

TREE CONTRACTION:

for Step $i$ from 1 to $n-2$

if $a(i)=1$ then node $a$ is removed

\section{TREE EXPANSION:}

for Step $i$ from $n-2$ downto 1

if $a(i)=1$ then node $a$ is added as the child of node $a$

Because the address of node $a$ in $E F C_{1}(n)$ has $n-2$ bits, each tree contraction (or tree expansion) takes $n-2$ steps.

THEOREM 9. The tree contraction (and tree expansion) algorithm for $T_{1}(n)$ takes $n-2$ steps and each step is conflict-free.

The proof for this theorem is straightforward, from the definition of $E F C$ and the tree contraction and expansion algorithms.

\section{EMBEDding}

This section shows how to emulate regular hypercubes on Extended Fibonacci Cubes. Then, we show that the Extended Fibonacci Cube can emulate binary trees more efficiently than the regular Fibonacci Cube, and almost as efficiently as the hypercube, even though the Extended Fibonacci Cube is a much sparser network than the hypercube.

THEOREM 10.

- $E F C_{1}(n)$ is a proper subgraph of $Q(n-2)$ when $n>4$.

- $Q(1)=E F C_{1}(3), Q(2)=E F C_{1}(4)$, and $Q(n)$ is a proper subgraph of $E F C_{1}(2 n)$ for $n>2$.

\section{THEOREM 11.}

- $E F C_{k}(n)$ is a proper subgraph of $Q(n-2)$ for $n \geq k+3$.

- $Q(k)=E F C_{k}(k+2), Q(k+1)=E F C_{k}(k+3)$, and $Q(n)$ is a proper subgraph of $E_{F C}(2 n-k+1)$ for $n>k+2$.

In graph embedding techniques, host and guest architectures are viewed as graphs $H$ and $G$, respectively, and then the graph $G$ is embedded into the graph $H$. In order to obtain efficient emulation of $G$ by $H$, various cost measures of an embedding must be optimized. Among them, the dilation of an edge of $G$ is defined as the length of the path onto which an edge of $G$ is mapped. The dilation of the embedding is the maximum edge dilation of $G$. The expansion of the embedding is the ratio of the number of nodes in $G$ to the number of nodes in $H$. The congestion of the embedding is the maximum number of paths containing an edge in $H$, where every path represents an edge in $G$. The load of an embedding is the maximum number of processors of $G$ assigned to any processor of $H$. In our study, we assume that the load of an embedding is restricted to one, i.e., the mapping is one-to-one. Intuitively, the dilation represents the maximum communication delay between the communicating nodes, the expansion is a measure of processor utilization, and the congestion measures maximum congestion along a link. Ideally, we would like to find embeddings with minimum dilation, expansion, and congestion.

A complete binary tree of height $n$, denoted by $B(n)$, has $2^{n+1}-1$ nodes. We have the following result that minimizes dilation without considering the expansion factor.

THEOREM 12. $B(n)$ can be embedded into $E F C_{1}(2 n+2)$ with dilation two and congestion two, and $B(n)$ can be embedded into $E F C_{1}(2 n+6)$ with dilation one and congestion one.

Clearly, the expansion of embedding $B(k)$ into $E F C_{1}(2 k+2)$ is $O\left(c^{k}\right)$ for some constant $1<c<2$. That is, the expansion increases drastically as $k$ increase.

Can we keep the expansion factor relatively stable without increasing dilation? The answer to this question is positive. Based on [4], the smallest Fibonacci Cube with at least $2^{k+1}-1$ nodes is $F C(t)$, where $t=1.47 k$. Since $\left|E F C_{1}(t+1)\right|=$ $2|F C(t)|$, the smallest Extended Fibonacci Cube will still be $E F C_{1}(t)$, where $t=1.47 k+c$. We will show that $B(2 k+4)$ can be embedded into $E F C_{1}(3 k+9)$ with dilation two. Note that, by ignoring the constant factor, 1.5 is the ratio of the dimension of $E F C_{1}$ to that of $B$, which is close to 1.47; therefore, the expansion grows slowly as $k$ increases. This can be shown using Table 2, where sizes of $E F C_{1}, F C, Q, B$ of various dimensions and levels are listed. For example, the expansion of embedding $B(6)$ into $E F C_{1}(12)$ is $178 / 127=1.402$ and the expansion of embedding $B(8)$ into $E F C_{1}(15)$ is $754 / 511=1.476$. Note that in Table 2 , symbol - represents an undefined entry.

We start with embedding of $n$-level double-rooted complete binary tree $D R C B(n)$ into the Extended Fibonacci Cube. The $\operatorname{DRCB}(n)$ tree is an $n$-level complete binary tree $B(n)$, with the root replaced by a path of length two. A $D R C B(n)$ contains $2^{n+1}$ nodes. The constructive proofs of Theorems 13 and 14 can be found in [15].

THEOREM 13. For any $m \geq 0, D R C B(2 m+4)$ can be embedded in $E F C_{1}(3 m+9)$ with dilation two and congestion two.

TABLE 2

SIZES OF $E F C_{1}, F C, Q, B$ OF VARIOUS DIMENSIONS AND LEVELS

\begin{tabular}{|c|c|c|c|c|c|c|c|c|c|c|c|c|c|c|c|}
\hline size $(n)$ & 1 & 2 & 3 & 4 & 5 & 6 & 7 & 8 & 9 & 10 & 11 & 12 & 13 & 14 & 15 \\
\hline$B(n)$ & 3 & 7 & 15 & 31 & 63 & 127 & 255 & 611 & 1,023 & 2,047 & 4,095 & 8,191 & 16,384 & 32,767 & 65,535 \\
\hline $\mathrm{Q}(\mathrm{n})$ & 2 & 4 & 8 & 16 & 32 & 64 & 128 & 255 & 612 & 1,024 & 2,048 & 4,096 & 8,192 & 16,384 & 32,768 \\
\hline $\mathrm{FC}(\mathrm{n})$ & 1 & 1 & 2 & 3 & 5 & 8 & 13 & 21 & 34 & 55 & 89 & 144 & 233 & 377 & 610 \\
\hline $\operatorname{EFC}_{1}(\mathrm{n})$ & - & - & 2 & 4 & 6 & 10 & 16 & 26 & 42 & 68 & 110 & 178 & 288 & 466 & 754 \\
\hline
\end{tabular}


Note that a complete binary tree $B(2 k+4)$ can be derived from $D R C B(2 k+4)$ by connecting a root node to the son of another root node. Since these two nodes are two distances apart, by carefully mapping two roots and their two sons [15] to avoid accumulative congestion and dilation, we can still embed $B(2 k+4)$ into $E F C_{1}(3 m+9)$ with dilation two and congestion two.

COROLLARY. For any $m \geq 0, B(2 k+4)$ can be embedded into $E F C_{1}(3 k+9)$ with dilation two and congestion two.

Similarly, the above results apply to $F C$.

THEOREM 14. For any $m \geq 0, D R C B(2 m+1)$ can be embedded in $F C(3 m+5)$ with dilation two and congestion two.

COROLLARY. For any $m \geq 0, B(2 k+4)$ can be embedded into $F C(3 k+9)$ with dilation two and congestion two.

Note that, in [5], it is shown that $B(2 n+1)$ can be embedded into $F C(3 k+5)$ with dilation three. Clearly, our result here is better which reduces dilation by one.

We compare regular hypercubes, Fibonacci Cubes, and the series of Extended Fibonacci Cubes in terms of degree of sparsity, network growth rate, and power of emulating other topologies. Let $F_{k}(n)$ and $L_{k}(n)$ denote the number of nodes and links in $\operatorname{EFC}_{k}(n)$, respectively, where $k \geq 1$.

THEOREM 15. For any $\operatorname{EFC}_{k}(n)$ with $n>k+3, F_{k}(n)=F_{k}(n-1)+$

$$
\begin{aligned}
& F_{k}(n-2), \text { where } F_{k}(k+2)=2^{k}, F_{k}(k+3)=2^{k+1} . L_{k}(n)= \\
& L_{k}(n-1)+L_{k}(n-2)+F_{k}(n-2), \text { where } L_{k}(k+1)=2^{k-1} * k, \\
& L_{k}(k+3)=2^{k} *(k+1) .
\end{aligned}
$$

COROLLARY. $F_{k}(n)=2^{k} * F(n-k), n \geq k+2$.

Two measures are used in comparison: The network growth rate is measured by the number of nodes in a given size $n$. The degree of sparsity is measured by the ratio of the number of links to the number of nodes in a network under consideration. The above equations can be plotted and results show that for Fibonacci Cubes, $E F C_{1}$, and $E F C_{2}$, both measures are very close with the rate (and ratio) for $E F C_{2}$ being slightly higher than the one for $E F C_{1}$, which, in turn, is slightly higher than the one for $F C$. Therefore, Fibonacci Cubes and Extended Fibonacci Cubes have the similar network growth rate and the degree of sparsity.

When a network is embedded in a (regular or Extended) Fibonacci Cube, the quality of embeddings can be measured by the ratio of the size of the embedded network to the size of the embedding cube (which is measured in terms of the number of nodes). This ratio reflects the utilization of nodes in the embedding cubes. Therefore, in general, the larger the ratio the better the embedding. Let $R_{G}(n)$ be the ratio of the size of the $n$-dimensional hypercube to the size of minimum embedding graph of type $G$.

THEOREM 16. For any size $n$,

$R_{F C}(n)<R_{E F C_{1}}(n)<R_{E F C_{2}}(n)<\ldots<R_{E F C_{k}}(n)<R_{E F C_{k+1}}(n)<\ldots$

The detailed results on network growth rates and degrees of sparcity for Extended Fibonacci Cubes can be found in [15].

\section{Conclusions}

In this paper, we have proposed a series of Extended Fibonacci Cubes which virtually maintains all the desirable properties of the Fibonacci Cube. In addition, Extended Fibonacci Cubes are Hamiltonian and they are better in terms of emulating other topologies than regular Fibonacci Cubes.

We believe that the series of Extended Fibonacci Cubes is a promising incomplete hypercube structure. This structure not only provides cube structures with arbitrary sizes, but also exposes the nature of hypercube systems operating in a gracefully degraded manner. For example, when some nodes become faulty in a hypercube $Q(n)$, we may try to determine a maximum $k(k<n-2)$, such that $E_{F} C_{k}(n)$ is a subgraph of the faulty hypercube. We envisage that the class of the Extended Fibonacci Cubes would be of interest to designers of cube-based parallel machines.

Possible future work includes, but not limited to:

1) Other possible extensions. One possible extension could be based on the $p$ th order Fibonacci numbers $[8]: F^{(p)}(n)=F^{(p)}(n-1)+F^{(p)}(n-2)+\ldots+F^{(p)}(n-p)$. By assigning appropriate initial conditions, a pth order Extended Fibonacci Cube is derived. Another possible extension, called Enhanced Fibonacci Cube [12], is based on a different recursive sequence: $F(n)=2 * F(n-2)+2$ * $F(n-4)$, whereas initial conditions $F(3)=2, F(4)=3$, $F(5)=5$, and $F(6)=8$. It has been shown that the Enhanced Fibonacci Cube is Hamiltonian and it maintains virtually all the desirable properties of the Fibonacci Cube. However, the relationship between the Extended and Enhanced Fibonacci Cubes is yet to be determined.

2) Reconfiguring a faulty hypercube into an Extended Fibonacci Cube $E F C_{k}$ with maximum $k$ is an interesting, but challenging, problem, the results of which will certainly provide insight into the nature of hypercube parallel machines operated in a gracefully degraded mode.

\section{REFERENCES}

[1] K. Abrahamson, N. Dadoun, D.G. Kirkpatrick, and T. Przxtycka, "A Simple Parallel Tree Contraction Algorithm," J. Algorithms, vol. 10, pp. 287-302, 1989.

[2] J. Bergum, B. Cong, and S. Sharma, "Simulation of Tree Structures on Fibonacci Cubes," Proc. First Int'l Conf. Computer Comm. and Networks, pp. 279-283, 1992.

[3] NCUBE 6400 Processor Manual. NCUBE Co., 1990.

[4] B. Cong and S.Q. Zheng, "Near-Optimal Embeddings of Trees into Fibonacci Cubes," Proc. 28th IEEE Southeastern Symp. System Theory, pp. 421-426, 1996.

[5] B. Cong, S.Q. Zheng, and S. Sharma, "On Simulations of Linear Arrays, Rings, and 2-D Meshes on Fibonacci Cube Networks," Proc. Seventh Int'l Parallel Processing Symp., pp. 748-751, 1993.

[6] R.L. Graham, D.E. Knuth, and O. Patashnik, "Special Numbers," Concrete Mathematics, chapter 6. Reading, Mass.: Addison-Wesley, 1989.

[7] J.P. Hayes, T.N. Mudge, Q.F. Stout, S. Colley, and J. Palmer, "A Microprocessor-Based Hybercube Supercomputer," IEEE Micro, vol. 6, no. 5, pp. 6-17, Oct. 1986.

[8] W.J. Hsu and M.J. Chung, "Generalized Fibonacci Cubes," Proc. 1993 Int'l Conf. Parallel Processing, vol. I, pp. 299-302, 1993.

[9] W.J. Hsu, C.V. Page, and J.S. Liu, "Computing Prefixes on a Large Family of Interconnection Topologies," Proc. 1992 Int'l Conf. Parallel Processing, vol. 3, pp. 153-159, 1992. 
[10] W.J. Hsu, "Fibonacci Cubes-A New Interconnection Topology," IEEE Trans. Parallel and Distributed Systems, vol. 4, no. 1, pp. 3-12, Jan. 1993.

[11] H.P. Katseff, "Incomplete Hypercubes," IEEE Trans. Computers, vol. 37, no. 5, pp. 604-608, May 1988.

[12] H. Qian and J. Wu, "Enhanced Fibonacci Cubes," Technical Report TR-CSE-94-16, Florida Atlantic Univ., Apr. 1994.

[13] Y. Saad and M.H. Schultz, "Topological Properties of Hypercubes," IEEE Trans. Computers, vol. 37, no. 7, pp. 867-872, July 1988.

[14] N.F. Tzeng, "Structural Properties of Incomplete Hypercube Computers," Proc. 10th IEEE Int'l Conf. Distributed Computing Systems, pp. 262-269, May 1990.

[15] J. Wu, "Extended Fibonacci Cubes," Technical Report TR-CSE-9362, Florida Atlantic Univ., Nov. 1993.

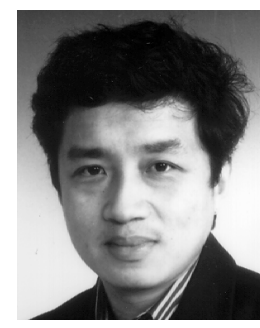

Jie $\mathbf{W u}$ received the $\mathrm{BS}$ degree in computer engineering in 1982, the MS degree in computer science in 1985, both from Shanghai University of Science and Technology, and the $\mathrm{PhD}$ degree in computer engineering from Florida Atlantic University, Boca Raton, Florida, in 1989. During 1985-1987, he taught at Shanghai University of Science and Technology. Since August 1989, he has been with the Department of Computer Science and Engineering, Florida Atlantic University, where he is an associate professor. Dr. Wu has published in IEEE Transactions on Software Engineering, IEEE Transactions on Computers, IEEE Transactions on Parallel and Distributed Systems, the Journal of Parallel and Distributed Computing, and Concurrency: Practice and Experience. His research interests are in the area of fault-tolerant computing, parallel/distributed algorithms, interconnection networks, Petri net applications, and software engineering. Dr. Wu serves on the editorial board of the International Journal on Computers and Applications. He has served on the program committees of the 1996 and 1998 IEEE International Conference on Distributed Computing Systems. He is a recipient of the $1996 \mathrm{Re}-$ searcher of the Year Award at Florida Atlantic University. Dr. Wu is a senior member of the IEEE and a member of the ACM. 